\title{
Black hole particle emission in higher-dimensional spacetimes
}

\author{
Vitor Cardosd* \\ Department of Physics and Astronomy, The University of Mississippi, University, MS 3867r-1848, USA ${ }^{\dagger}$ \\ Marco Cavaglia羽 \\ Department of Physics and Astronomy, The University of Mississippi, University, MS 38677-1848, USA \\ Leonardo Gualtier 8 \\ Centro Studi e Ricerche E. Fermi, Compendio Viminale, 00184 Rome, Italy and \\ Dipartimento di Fisica Università di Roma "La Sapienza"/Sezione INFN Roma1, Piazzale Aldo Moro 2, 00185 Rome, Italy

\begin{abstract}
In models with extra dimensions, a black hole evaporates both in the bulk and on the visible
\end{abstract} \\ brane, where standard model fields live. The exact emissivities of each particle species are needed \\ to determine how the black hole decay proceeds. We compute and discuss the absorption cross- \\ sections, the relative emissivities and the total power output of all known fields in the evaporation \\ phase. Graviton emissivity is highly enhanced as the spacetime dimensionality increases. Therefore, \\ a black hole loses a significant fraction of its mass in the bulk. This result has important consequences \\ for the phenomenology of black holes in models with extra dimensions and black hole detection in \\ particle colliders. \\ PACS numbers:
}

A black hole is a grey body with temperature proportional to its surface gravity [1]. The black hole emission spectrum depends crucially on the structure and dimensionality of the embedding spacetime. In models with large [2] or warped [3] extra dimensions, standard model fields, except the graviton, are constrained to propagate on a four-dimensional submanifold of the higher-dimensional spacetime. In this scenario, the relative emissivities of the fields (greybody factors) are essential to determine if the black hole evaporates mainly on the four-dimensional brane or in the higher-dimensional bulk. This is particularly important for models of lowenergy scale gravity, where detection of subatomic black holes in particle colliders and ultrahigh-energy cosmic ray observatories is only possible if a consistent fraction of the initial black hole mass is channeled into brane fields [4]. Counting of brane vs. bulk degrees of freedom (d.o.f.) provides a naive argument in support of the dominance of brane over bulk emission [5]: Since all standard model fields carry a larger number of d.o.f. than the graviton, brane emission must dominate over bulk emission. However, a large emissivity for bulk fields could invalidate this conclusion [6]. If the probability of emitting spin-2 quanta is much higher than the probability of emitting lower spin quanta, the black hole may evaporate mainly in the bulk.

The relative emissivities per d.o.f. of a fourdimensional non-rotating black hole are 1, 0.37, 0.11 and 0.01 for spin- $0,-1 / 2,-1$ and -2 , respectively [7]. In that case, the graviton power loss is negligible compared to

\footnotetext{
${ }^{\dagger}$ Also at Centro de Física Computacional, Universidade de Coimbra, P-3004-516 Coimbra, Portugal
}

the loss in other standard model channels. Since brane fields are constrained in four dimensions, the relative greybody factors (relative emissivities) for these fields are expected to approximately retain the above values in higher dimensions. The graviton emission is expected to be larger due to the increase in the number of its helicity states. The helicity states of a massless particle in $D$-dimensions are given by the representation of the little group $S O(D-2)$, i.e. the group of spatial rotations preserving the particle direction of motion. For instance, a five-dimensional graviton has five helicity states, corresponding to the $S O(3)$ group of orthogonal rotations to the direction of motion.

Therefore, a conclusive statement on brane vs. bulk emission rates requires the knowledge of the greybody factors for all fields. The higher-dimensional emission rates for spin- $0,-1 / 2$ and -1 fields are known [8]. However, the exact emission rate for spin-2 fields has not been computed yet. (See, however, Ref. [9]). In this paper we fill this gap. We find that the graviton emissivity is highly enhanced as the spacetime dimensionality increases. Although this increase is not sufficient to lead to a domination of bulk emission over brane emission, a consistent fraction of the higher-dimensional black hole mass is lost in the bulk. This fact has important consequences for the phenomenology of black hole events in low-scale gravity and primordial black hole formation.

The $D$-dimensional non-rotating black hole is described by the higher-dimensional Schwarzschild metric, also known as Tangherlini metric [10]

$$
d s^{2}=-f d t^{2}+f^{-1} d r^{2}+r^{2} d \Omega_{D-2}^{2}, \quad f=1-\frac{1}{r^{D-3}},
$$

where $d \Omega_{D-2}^{2}$ is the line element on the unit sphere $S^{D-2}$ and the black hole radius has been set to $r_{H}=1$ without 
loss of generality. The formalism to handle gravitational perturbations of the Tangherlini metric has been developed by Kodama and Ishibashi 11 following previous work in four dimensions by Regge and Wheeler 12] and Zerilli [13. There are three kinds of gravitational perturbations: scalar, vector and tensor. Scalar and vector perturbations have their counterparts in $D=4$. The tensor type appears only in higher dimensions. The evolution equation for the perturbations is

$$
\frac{d^{2} \Psi}{d r_{*}^{2}}+\left(\omega^{2}-V\right) \Psi=0
$$

where $r$ is a function of the tortoise coordinate $r_{*}$ defined by $\partial r / \partial r_{*}=f$. For vector and tensor perturbations, the potential $V$ is

$V=f\left[\frac{l(l+D-3)}{r^{2}}+\frac{(D-2)(D-4)}{4 r^{2}}+\frac{\left(1-p^{2}\right)(D-2)^{2}}{4 r^{D-1}}\right]$

where $l \geq 2,3, \ldots$ is the angular quantum number and $p=2$ (0) for vector (tensor) perturbations, respectively. For scalar perturbations, the potential is

$$
V=f \frac{Q(r)}{16 r^{2} H(r)^{2}}
$$

$$
\begin{aligned}
Q(r) & =(D-2)^{4}(D-1)^{2} x^{3}+(D-2)(D-1)\left\{4\left[2(D-2)^{2}-3(D-2)+4\right] m+(D-2)(D-4)(D-6)(D-1)\right\} x^{2} \\
& -12(D-2)[(D-6) m+(D-2)(D-1)(D-4)] m x+16 m^{3}+4 D(D-2) m^{2}, \\
H(r) & =m+\frac{1}{2}(D-2)(D-1) x, \quad m=l(l+D-3)-(D-2), \quad x=1 / r^{D-3} .
\end{aligned}
$$

Assuming a harmonic wave $e^{i \omega t}$ and ingoing waves near the horizon, we have the following boundary condition:

$$
\Psi(r) \rightarrow e^{-i \omega r_{*}} \sim(r-1)^{-i \omega /(D-3)}, \quad r \rightarrow r_{+} .
$$

For large $r_{*}$ we have both out- and in-going waves:

$$
\Psi(r) \rightarrow T e^{-i \omega r_{*}}+R e^{i \omega r_{*}}, \quad r_{*} \rightarrow \infty .
$$

The absorption probability for the wave is $|\mathcal{A}|^{2}=\left(|T|^{2}-\right.$ $\left.|R|^{2}\right) /|T|^{2}$. The number of absorbed particles is

$$
\frac{d N_{a b s}}{d t}=\sigma \Phi
$$

where $\Phi$ is the flux and $\sigma$ is the absorption cross-section. A sum over all final states and an average over initial states is understood. Solving the equations for the gravitational perturbations with an expansion in spherical harmonics, we find

$$
\sigma=C(D, \omega) \sum_{l}\left[N_{l S}\left|\mathcal{A}_{l S}\right|^{2}+N_{l V}\left|\mathcal{A}_{l V}\right|^{2}+N_{l T}\left|\mathcal{A}_{l T}\right|^{2}\right]
$$

where the subscripts $S, V$ and $T$ refer to scalar, vector and tensor perturbations, respectively. The multiplicities $N_{l}$ are defined in Ref. 14] and the normalization is $C(D, \omega)=2(2 \pi / \omega)^{D-2}\left[D(D-3) \Omega_{D-2}\right]^{-1}$.

The wave equation, Eq. (2), can be solved in the lowenergy regime $\omega \ll 1$. (For details, see Ref. [15].) This method uses a matching procedure to find a solution valid throughout the whole spacetime for any value of $p$. Low frequencies give a substantial contribution to the total Hawking power emission, thus providing a good approximation of the exact result. The absorption probability is

$$
|\mathcal{A}|^{2}=1-|\mathcal{R}|^{2}=4 \pi\left(\frac{\omega}{2}\right)^{D+2 l-2} \frac{\Gamma\left(1+\frac{2 l+p(D-2)}{2(D-3)}\right)^{2} \Gamma\left(1+\frac{2 l-p(D-2)}{2(D-3)}\right)^{2}}{\Gamma\left(1+\frac{2 l}{D-3}\right)^{2} \Gamma\left(l+\frac{(D-1)}{2}\right)^{2}} .
$$

The low-energy absorption probability for spin-0 fields 16 and spin-2 tensor perturbations is recovered by setting $p=0$ in the above equation. The result for vector perturbations is obtained by setting $p=2$. Gravita- tional scalar perturbations cannot be dealt with analytically. However, numerical simulations give an effective $p_{\text {grav scalar }} \sim 2+0.674 D^{-0.5445}$. The low-energy absorption cross section can be obtained from Eqs. (11). For 
instance, in four-dimensions, where the tensor contribution disappears, the $l=2$ mode gives the cross section $\sigma_{l=2}=4 \pi \omega^{4} / 45$. This result agrees with the well-known result of Ref. [7].

The wave equation can also be solved in the highenergy limit. In that case, the absorption cross section is expected to approximate the cross section for particle capture. This conjecture has been verified in a number of papers for the scalar field. (See Ref. [8] and references therein.) The proof for spin-1 and spin-2 fields is sketched below. (For further details, see Ref. [15].)
Since high frequencies can easily penetrate the gravitational potential barrier, the absorption probabilities of all fields approximate 1 as $\omega \rightarrow \infty$. The cross section in the high-energy limit must include the contribution from all $l \lesssim \omega$. Therefore, the largest contribution to the cross section is given by high- $l$ modes. Since the high- $l$ limit of the wave equation is independent of the type of perturbation [17], it follows $\mathcal{A}_{l S}=\mathcal{A}_{l V}=\mathcal{A}_{l T}$. The universality of the high-energy absorption cross section follows from Eq. (10) and the properties of the multiplicities.

The total energy flux for the gravitational radiation is

$$
\frac{d E}{d t}=\frac{d E_{S}}{d t}+\frac{d E_{V}}{d t}+\frac{d E_{T}}{d t}=\sum_{l} \int \frac{d \omega}{2 \pi} \frac{\omega}{e^{\omega / T_{H}}-1}\left(N_{l S}\left|\mathcal{A}_{l S}^{s=2}\right|^{2}+N_{l V}\left|\mathcal{A}_{l V}^{s=2}\right|^{2}+N_{l T}\left|\mathcal{A}_{l T}^{s=2}\right|^{2}\right),
$$

where we have separated the individual contributions of each harmonics. The Hawking temperature is $T_{H}=(D-$ $3) /(4 \pi)$. Note that the number of helicities is included in the multiplicity factors and the sum of scalar, vector and tensor contributions.

The absorption probabilities $\mathcal{A}$ for all frequencies can be computed numerically. Equation (3) is integrated from a point near the horizon (typically $r-1 \sim 10^{-6}$ ), where the field behavior is given by Eq. (7). The numerical result is compared to Eq. (8) at large $r$. A better accuracy is achieved by considering the next-to-leading order correction term (see Ref. [18])

$\Psi(r) \rightarrow T\left(1+\frac{\varepsilon}{r}\right) e^{-i \omega r_{*}}+R\left(1-\frac{\varepsilon}{r}\right) e^{i \omega r_{*}}, \quad r_{*} \rightarrow \infty$.

This allows the determination of the coefficients $T, R$ and the absorption probability $\mathcal{A}$. The results for the total integrated power, Eq. (12), are summarized in Table [ The values for the lower-spin fields living on the brane are taken from Ref. [8]. The graviton values are normalized to the four-dimensional case, where $P=1.52 \times 10^{-5}$. This is in exact agreement with Page's result. (See Table I of Ref. 7].) The results for lower-spin fields are normalized to their four-dimensional values. It is worth discussing some features of the numerical results: (i) The relative contribution of the higher partial waves increases with $D$. For instance, in four dimensions the contribution of the $l=2$ mode is two orders of magnitude larger than the contribution of the $l=3$ mode. More energy is channeled in $l=3$ mode than in $l=2$ mode for $D \geq 9$. (The largest tensor contribution in ten dimensions comes from the $l=4$ mode.) Contributions from high $l$ are needed to obtain accurate results for large $D$. For instance, in ten dimensions the first 10 modes must be considered for a meaningful result. The values in Table \ have a $5 \%$ accuracy. (ii) The total power radiated in gravitons increases more rapidly than the power radiated in lowerspin fields as $D$ increases. This is due to the increase in the multiplicity of the tensor perturbations, which is larger than the scalar multiplicity by a factor $D^{2}$ at high $D$. Therefore, the main contribution to the total power comes from the tensor (and vector) modes. For instance, in ten dimensions the tensor mode contributes roughly half of the total power output.

Table II gives the fraction of radiated power per d.o.f. normalized to the scalar field, where the graviton value includes all the helicity states. In four dimensions, the power loss in gravitons is negligible compared to the power loss in lower-spin fields. The graviton channel is only about $5 \%$ of the scalar channel. This conclusion is reversed in higher dimensions. For instance, the graviton loss is about 35 times higher than the scalar loss in $D=11$. Although the graviton emission is highly enhanced, the large number of brane d.o.f. (more than 100 for the standard model) assures that the brane channel dominates on the bulk channel. However, power loss in the bulk is significant and cannot be neglected at high $D$; about $1 / 4$ of the initial black hole mass is lost in the 11-dimensional bulk. The particle emission rates per d.o.f. are shown in Table III. The relative emission rates of different fields can be obtained by summing on the brane d.o.f. For instance, the relative emission rates of standard model charged leptons (12 d.o.f.) and the 11-dimensional bulk graviton are roughly 1:1. This ratio becomes $\sim 40: 1$ in five dimensions. We find again that the bulk energy loss is significant in higherdimensional spacetimes. This long overdue computation is of paramount importance for the phenomenology of any higher-dimensional gravitational models. Our main result is that the power loss in the graviton channel is highly enhanced in higher-dimensional spacetimes. This has important consequences for the detection of mi- 
TABLE I: Total power $P$ of Hawking radiation channeled into different fields. The first three rows correspond to fields propagating on the brane. The last row is the power radiated in bulk gravitons normalized to the four-dimensional case.

\begin{tabular}{ccccccccc}
\hline \hline$D$ & 4 & 5 & 6 & 7 & 8 & 9 & 11 \\
\hline Scalars & 1 & 8.94 & 36 & 99.8 & 222 & 429 & 749 \\
Fermions & 1 & 14.2 & 59.5 & 162 & 352 & 664 & 1140 \\
Gauge Bosons & 1 & 27.1 & 144 & 441 & 1020 & 2000 & 3530 \\
Gravitons & 1 & 103 & 1036 & 5121 & $2 \times 10^{4}$ & $7.1 \times 10^{4}$ & $2.5 \times 10^{5}$ & $8 \times 10^{5}$ \\
\hline \hline
\end{tabular}

TABLE II: Fraction of radiated power per d.o.f. normalized to the scalar field. The graviton d.o.f. (number of helicity states) are included in the results.

\begin{tabular}{ccccccccc}
\hline \hline$D$ & 4 & 5 & 6 & 7 & 8 & 9 & 10 & 11 \\
\hline Scalars & 1 & 1 & 1 & 1 & 1 & 1 & 1 & 1 \\
Fermions & 0.55 & 0.87 & 0.91 & 0.89 & 0.87 & 0.85 & 0.84 & 0.82 \\
Gauge Bosons & 0.23 & 0.69 & 0.91 & 1.0 & 1.04 & 1.06 & 1.06 & 1.07 \\
Gravitons & 0.053 & 0.61 & 1.5 & 2.7 & 4.8 & 8.8 & 17.7 & 34.7 \\
\hline \hline
\end{tabular}

TABLE III: Fraction of emission rates per d.o.f. normalized to the scalar field. The graviton result includes all the helicity states and counts as one d.o.f.

\begin{tabular}{ccccccccc}
\hline \hline$D$ & 4 & 5 & 6 & 7 & 8 & 9 & 10 & 11 \\
\hline Scalars & 1 & 1 & 1 & 1 & 1 & 1 & 1 & 1 \\
Fermions & 0.37 & 0.7 & 0.77 & 0.78 & 0.76 & 0.74 & 0.73 & 0.71 \\
Gauge Bosons & 0.11 & 0.45 & 0.69 & 0.83 & 0.91 & 0.96 & 0.99 & 1.01 \\
Gravitons & 0.02 & 0.2 & 0.6 & 0.91 & 1.9 & 2.5 & 5.1 & 7.6 \\
\hline \hline
\end{tabular}

croscopic black hole formation in particle colliders and ultrahigh-energy cosmic ray observatories, where a larger bulk emission implies larger missing energies and lower multiplicity in the visible channels. Despite the increase in graviton emissivity, a non-rotating black hole in the Schwarzschild phase will emit mostly on the brane due to the higher number of brane d.o.f. However, black hole energy loss in the bulk cannot be neglected in presence of extra dimensions.

The effects of graviton emission may be even more dramatic when one includes rotation. Consider a rotating four-dimensional black hole [19]. Graviton emission, which is supressed for small rotations, rapidly increases with the angular momentum $J$. (In four dimensions $J$ ranges from 0 to $M^{2}$ ). As $J$ grows from 0 to $0.7 M^{2}$ graviton emissivity grows 3 orders of magnitude (see Table I in Ref. 19]) while the emissivities of fermion and gauge bosons grow less than one order of magnitude. A similar behavior is expected in higher dimensions, where for $D>5$ there is no upper bound on $J$, implying that graviton emission dominates the evaporation process. Since known results for rotating black holes in $D$-dimensions do not include gravitons [20], this remains an open question.

Acknowledgements. We are very grateful to De-
Chang Dai for pointing out some typos in previous versions. We would like to thank Akihiro Ishibashi for useful correspondence. VC acknowledges financial support from FCT through PRAXIS XXI program, and from Fundação Calouste Gulbenkian.

* Electronic address: vcardoso@phy.olemiss.edu

¥ Electronic address: cavaglia@phy.olemiss.edu

$\S$ Electronic address: gualtieri@roma1.infn.it

[1] S. W. Hawking, Commun. Math. Phys. 43, 199 (1975).

[2] N. Arkani-Hamed, S. Dimopoulos and G. R. Dvali, Phys. Lett. B 429, 263 (1998); I. Antoniadis et al. Phys. Lett. B 436, 257 (1998).

[3] L. Randall and R. Sundrum, Phys. Rev. Lett. 83, 3370 (1999); Phys. Rev. Lett. 83, 4690 (1999).

[4] M. Cavaglià, Int. J. Mod. Phys. A 18, 1843 (2003); M. Cavaglià, S. Das and R. Maartens, Class. Quant. Grav. 20, L205 (2003); V.P. Frolov and D. Stojkovic, Phys. Rev. Lett. 89, 151302 (2002)

[5] R. Emparan, G. T. Horowitz and R. C. Myers, Phys. Rev. Lett. 85, 499 (2000).

[6] M. Cavaglià, Phys. Lett. B 569, 7 (2003).

[7] D.N. Page, Phys. Rev. D 13, 198 (1976).

[8] C.M. Harris and P. Kanti, JHEP 0310, 014 (2003); P. Kanti, Int. J. Mod. Phys. A 19, 4899 (2004).

[9] A.S. Cornell, W. Naylor, M. Sasaki, hep-th/0510009

[10] F.R. Tangherlini, Nuovo Cim. 27, 636 (1963); R. C. Myers and M.J. Perry, Ann. Phys. 172, 304 (1986).

[11] H. Kodama and A. Ishibashi, Prog. Theor. Phys. 110, 701 (2003); ibid. 110, 901 (2003); ibid. 111, 29 (2004).

[12] T. Regge, J.A. Wheeler, Phys. Rev. 108, 1063 (1957).

[13] F. Zerilli, Phys. Rev. Lett. 24, 737 (1970).

[14] A. Chodos and E. Myers, Annals of Physics (NY) 156, 412 (1984); M.A. Rubin and C.R. Ordóñez, J. Math. Phys. 25, 2888 (1984); J. Math. Phys. 26, 65 (1985).

[15] V. Cardoso, M. Cavaglià, and L. Gualtieri, JHEP02, 021 (2006).

[16] P. Kanti and J. March-Russell, Phys. Rev. D 66, 024023 (2002).

[17] V. Cardoso, O.J. Dias, and J.P. Lemos, Phys. Rev. D 67, 064026 (2003).

[18] E. Berti, M. Cavaglià and L. Gualtieri, Phys. Rev. D 69, 124011 (2004).

[19] D.N. Page, Phys. Rev. D 14, 3260 (1976).

[20] D. Ida, K.Y. Oda and S.C. Park, Phys. Rev. D 71, 124039 (2005); E. Jung, S.H. Kim and D.K. Park, Phys. Lett. B 615, 273 (2005); M. Casals, P. Kanti and E. Winstanley, hep-th/0511163 